DOSSIÊ TEMÁTICO: A medicalização da educação no Brasil e no Chile: diferentes perspectivas

do https://doi.org/10.22481/praxisedu.v15i36.5862

\title{
EXCESO DE ATENCIÓN: DE LA COMPOSICIÓN DE UN TRASTORNO EN LA ESCUELA CHILENA
}

\author{
EXCESS OF ATTENTION: THE CONSTRUCTION OF A DISORDER IN CHILEAN \\ SCHOOLS
}

EXCESSO DE ATENÇÃO: A COMPOSIÇÃO DE UM TRASTORNO NA ESCOLA CHILENA

\author{
Hugo Sir \\ Universidad de Chile - Chile \\ Université Paris 8 - França \\ Isis Castañeda \\ Universidad de Chile - Chile \\ Esteban Radiszcz \\ Universidad de Chile - Chile
}

\begin{abstract}
Resumen: El presente artículo tiene por objetivo explorar las condiciones epistémicas y prácticas de producción del diagnóstico de TDAH en la escuela en Chile. Se desarrolla en dos ejes: 1) la posición de la escuela en Chile respecto de las características y tratamiento de esta categoría diagnóstica; 2) la construcción del diagnóstico en el aula, analizando el tratamiento del comportamiento atencional y la configuración de la situación TDAH. Concluiremos situando la interrogante sobre la inquietud escolar en torno al diagnóstico como indicio de un choque de órdenes civilizatorios expresado en las formas de modulaciones afectivas en el aula, donde se buscará resolver a nivel de una exigencia de rentabilización individual violencias institucionales.
\end{abstract}

Palabras clave: Atención. Escuela en Chile. Situación TDAH.

\begin{abstract}
The aim of this article is to explore the epistemic and practical conditions of production of the diagnosis of ADHD in Chilean schools. It develops along two main lines: 1) the role of schools in Chile in relation to the characteristics and treatment of this diagnostic category; and 2) the construction of the diagnosis in the classroom, analyzing the treatment of attentional behavior and the configuration of the ADHD Situation. We will conclude by considering student anxiety related to the diagnosis as an indicator of a clash of civilizational orders expressed in the modes of affective modulation performed in the classroom, wherein attempts will be made to resolve institutional violence by way of a demand for individual reform.
\end{abstract}

Keywords: Attention. Chilean school. ADHD Situation.

Resumo: O objetivo deste artigo é explorar as condições epistêmicas e as práticas de produção do diagnóstico de TDAH nas escolas do Chile. Se desenvolve em dois eixos: 1) A posição da escola no 
Chile em relação às características e tratamento dessa categoria diagnóstica; e 2) a construção do diagnóstico em sala de aula, analisando o tratamento do comportamento atencional e a configuração da situação-TDAH. Concluímos situando a questão da inquietude escolar em torno do diagnóstico como uma indicação de um choque de ordens civilizatórias expressas nas formas de modulações afetivas na sala de aula, onde a violência institucional resolve-se através de uma exigência de rentabilização individual.

Palavras chave: Atenção. Escola no Chile. Situação-TDAH.

\section{Introducción}

El diagnóstico conocido como Trastorno por Déficit de Atención e Hiperactividad (TDAH), debe el comienzo de esta formulación a la 3a edición del Diagnostic and Statistical Manual of Mental Disorders (DSM-III) que data de 1980 (AMERICAN PSYCHIATRIC ASSOCIATION, 1980) y ya desde ahí apareció como una categoría particularmente controvertida (PARENS; JOHNSTON, 2009; SINGH, 2008; STIGLITZ, 2006). Consecuentemente, en Chile al igual que otras latitudes su entrada se ha considerado posterior a esa fecha y, por tanto, siendo de algún modo un diagnóstico importado (ROJAS et al., 2018), con escasas referencias a las condiciones epistémicas que delimitan su recepción. Sin que podamos desarrollar con profundidad esta arista en el presente trabajo parece, no obstante, relevante comenzar desde un lugar divergente para comprender la prevalencia, las condiciones y las singulares características de este diagnóstico en Chile.

En 1973, año por lo demás nada indiferente para el país en cuestión, Bravo edita un libro titulado "Trastornos del aprendizaje y de la conducta escolar. Psicopatología de la disfunción cerebral infantil" (BRAVO, 1977). Su intención es delimitar de la manera más acuciosa posible, con el conocimiento científico más asentado, el diagnóstico que precede al actual TDAH, la disfunción cerebral mínima. Encontraremos aquí una serie de elementos que veremos repetirse hasta nuestros días incansablemente en la discusión sobre las características de la(o)s niña(o)s que pueden ser catalogados con esta etiqueta. Particularmente relevante nos parecerá aquí que ya respecto de aquella descripción nosográfica nuestro autor señala la importancia de la escuela, única instancia que revela estas conductas como problemáticas. Es porque las principales consultas en psiquiatría infantil en aquel tiempo y a gran escala refieren a este tipo de problemas conductuales y de aprendizaje, que se requiere una descripción científica que permita deslindarlo de otros problemas conocidos con anterioridad, como por ejemplo, la “deficiencia mental leve o inmadurez pedagógica” (BRAVO, 1977, p.15) y, a reglón seguido, explicita que el abordaje de este síndrome: 
por su naturaleza misma, comprende aspectos neurológicos, psicológicos, psiquiátricos, pedagógicos y sociales que dificultan más aún su diagnóstico oportuno, pues exige un enfoque interdisciplinario integrado que no es posible realizar siempre en la práctica pedagógica o clínica de cada día. (BRAVO, 1977, p.15).

Dos antecedentes residen en este elemento arqueológico, entendido como una pista de aquello que, atravesando diversos campos del saber formalmente divididos, les otorga cierta inclinación de conjunto, cierto número de problemas que los conectan muchas veces de manera subrepticia (FOUCAULT, 1993; RODRÍGUEZ, 2019) y, con ello, nos permite aproximarnos de un modo diverso a la constitución de los objetos que definirán el campo de sus saberes, en este caso, la zona fronteriza entre lo neuropsiquiátrico y lo educativo. Por una parte, el diagnóstico se describe como estrictamente escolar, es decir, quizás no sería problemático si no hubiese ciertas exigencias específicas de esta institución. Por otra, que, en tanto eso es así, entonces la definición y gestión de éste implica, antes incluso que se convierta en vulgata de los departamentos universitarios en busca de fondos, una aproximación inter o transdisciplinaria.

El cuadro, nos dice Bravo, con una actualidad de más de 50 años "hunde sus raíces en la neurología y prolonga sus consecuencias a la pedagogía" (BRAVO, 1977, p.16). Y, sin embargo aún más, orgulloso de una escritura basada en la investigación empírica ${ }^{1}$, nos dirá en la introducción a la segunda edición que la principal diferencia con la primera proviene de la experiencia educacional que ante niño(a)s con trastornos específicos de aprendizaje "no tenían profesores especializados que los rehabilitara", cuestión que lo lleva a redefinir la especificidad de su investigación hacia la conducta escolar y específicamente a "algunas investigaciones neuropsicológicas que permitan comprender mejor las relaciones entre el síndrome de la disfunción cerebral mínima y los problemas escolares" (BRAVO, 1977, p.19).

¿Qué es entonces, desde aquí, el problema con la escuela y, con ello, parte de las condiciones epistémicas de recepción del diagnóstico oficializado en 80 con otra rúbrica? Pues bien, cuestiones bastante conocidas en cualquier descripción (hegemónica) contemporánea del TDAH: comportamientos disruptivos, impulsivos, peligros de que estos deriven en conductas delictuales o adictivas, incapacidad para aquietarse y cumplir las exigencias escolares, en definitiva, una serie de problemas que darían cuenta de dificultades adaptativas. Ahora bien, una nota precisa nos permitirá conectar con aquello que buscamos desarrollar en este artículo.

\footnotetext{
${ }^{1}$ Investigación que nos señala el autor se inicia con un equipo ya desde 1964 en el Hospital Luis Calvo Mackenna, lugar que hasta el día de hoy será relevante en la investigación sobre TDAH en niñas y niños chilenos.
} 
Nos dice el autor que la impulsividad y agresividad, en apariencia desproporcionada frente al ambiente, si bien "guarda relación con la situación ambiental [referido al "ambiente familiar escolar"] y el nivel de tensión emocional preexistente (...) también aparece como resultado de una mayor 'instintividad' (sic) en el comportamiento" (BRAVO, 1977, p.127). La intención expresada abiertamente por el autor es, a través de sus investigaciones, dar a conocer que estas problemáticas expresadas en y por la escuela no hablan de niños "tontos" o "flojos", sino que de asuntos que se hundirían en los cerebros infantiles, o sea, en los cerebros humanos y, en tanto eso es así, serían tratables. ¿No resuena acaso en esta instintividad cerebral algo necesario de colonizar (BURMAN, 2019), de civilizar (ELIAS, 1990, 1998), la necesidad una vez más de una sabia conducción de los afectos (LARA et al., 2017), esta vez fundada en una biología?

El vínculo entre TDAH y exigencias civilizatorias ha sido sugerido antes, aunque no ha sido consecuentemente desarrollado (CALIMAN, 2008, 2012; LAKOFF, 2000). No obstante, nos parece de alta relevancia a la hora de comprender aquello que, mediante el diagnóstico, se busca problematizar en Chile. Efectivamente, a través de los modos en que se ensamblan las categorías de salud mental es posible acercarse también a formas de poner en común inquietudes de órdenes que exceden la rigurosidad médica de éstos (EHRENBERG, 2010). En ese sentido la alta prevalencia que el diagnóstico muestra en el país, triplicando las tasas comúnmente observadas en el ámbito internacional (DE LA BARRA et al., 2012, THOMAS et al., 2015) $)^{2}$, además de sus características peculiares, por ejemplo: i) el veloz salto en su prevalencia entre los años 2009 y 2013 (Ministerio de Educación (MINEDUC), 2009c; Ministerio de Salud (MINSAL), 2013), cuestión que se refleja, en las importaciones chilenas de Metilfenidato $\left(\right.$ Ritalín $^{\circledR}$ ) que, entre 2006 y 2017, se incrementaron en un 450\% (CÁMARA DE DIPUTADOS DE CHILE, 2019); y ii) el aparente predominio de la dimensión atencional que primaría en el modo de describirse, delimitarse y gestionarse el diagnóstico en Chile (BERGEY et al., 2018), invitan a hacer partícipe aquellos otros campos sociales y disciplinas que delimitan la visibilidad singular del objeto en cuestión, es decir, la episteme (FOUCAULT, 1993) en donde este no solo adquiere existencia, sino en la cual parece poder connotar preocupaciones que le desbordan como categoría de salud y, por tanto, nos permitirían comprender el modo en que se vincula con problemas, en apariencia, específicamente escolares.

\footnotetext{
${ }^{2}$ Alcanzando en niña(o)s de entre 4 y 11 años un promedio del 15,5\%, como señalan (VICENTE, LA BARRA et al., 2012) Mientras que a nivel internacional éstas rodean el 5 o $6 \%$, alcanzando un $11 \%$ solo en EEUU, respecto del cual el TDAH se consideró una enfermedad cultural hasta 1980. Ver: (BERGEY et al., 2018; CONRAD; BERGEY, 2014; WENDER; TOMB, 2017; STIGLITZ, 2006).
} 
Con tales objetivos, entonces, el artículo se desarrollará de la siguiente forma. En primer lugar, daremos cuenta de la metodología de la investigación colectiva llevada a cabo por el equipo del Laboratorio Transdisciplinar en Prácticas Sociales y Subjetividad (LaPSoS) de la Facultad de Ciencias Sociales de la Universidad de Chile, que está detrás de estas reflexiones, siendo en efecto esta publicación parte de los resultados de más de 3 años de trabajo ${ }^{3}$. En segundo lugar, al estar vinculado el diagnóstico, antes incluso de su actual formulación, a un problema en la escuela, se nos impone revisar qué de particular tiene la posición de ésta en Chile que se traduce en las singulares características de esta categoría nosográfica en nuestro país. En tercer lugar, buscaremos plantear una lectura del modo en que se construye el diagnóstico desde el interior de las salas de clase y cómo en éstas se traduce la tensión que genera la posición de la escuela en Chile como una atención sobre la escuela, desplazándose como una atención sobre la atención en el aula, individualizando y sustancializando el problema. Así, finalmente, exploraremos cómo esto puede dar indicios de un choque de órdenes civilizatorios expresados en una dimensión fundamental para este proceso: las modulaciones afectivas en las relaciones entre generaciones (ELIAS, 1998). A la vez que, al buscar resolver a nivel de los individuos violencias institucionales (FASSIN, 2016), puede conducir a un bloqueo de transformaciones que, en tanto insisten por desplegarse, por modificar las instituciones, aparecen como persistente malestar (ROLNIK, 2019) para las escuelas y sus actores involucrados.

\section{Metodología}

La investigación colectiva se inicia en 2017 con el proyecto titulado "Trayectorias de malestar en niños(as) y adultos(as) diagnosticados(as) con TDAH: experiencia subjetiva y social de un sufrimiento multiforme", condecido por el Laboratorio Transdisciplinar en Prácticas Sociales y Subjetividad (LaPSoS) perteneciente a la Facultad de Ciencias Sociales de la Universidad de Chile. A través de esta vía de financiamiento se ha logrado dar forma a un trabajo empírico que, aun en desarrollo, contempla hasta la fecha:

\footnotetext{
${ }^{3}$ Aprovechamos pues de agradecer a equipo en cuyo seno todas las reflexiones aquí expresadas han emergido y, como buen trabajo colectivo, ya no sabemos con certeza a quien pertenecen cada una de las ideas, lo cual nos parece de todas formas una bendición. Por cierto, en tanto somos nosotro(a)s quienes las expresamos aquí, nos hacemos responsable de cualquier descuido u omisión, que no es otra cosa que el modo en que el conocimiento aparece antes que como búsqueda de la verdad, como un mapeo constante de aquello que falla y que, por tanto, nos permitiría a posteriori errar mejor.
} 
- tres etnografías breves, de 3 a 4 meses de duración, en tres establecimientos educacionales con características divergentes: a) colegio municipal en una comuna de clase media/media-alta, al que asisten, sin embargo, una gran cantidad de hija(o)s de trabajadora(e)s de la comuna; b) colegio semi-privado ${ }^{4}$ en una comuna de clase media/media baja; c) colegio privado en una comuna de clase alta $^{5}$.

- 23 entrevistas tanto con mujeres y hombres adultos como con niños y niñas, los(as) cuales habían sido diagnosticados(as) y/o tratados(as) por TDAH en algún momento de sus vidas.

- 7 entrevistas abiertas a niñas, niños y sus padres y/o cuidadores principales. 4 niños y 3 niñas de entre 7 y 13 años, contactados a partir de la observación etnográfica y habiendo recibido diagnóstico y tratamiento por TDAH.

- 7 a profesionales de la salud mental.

- 4 grupos de discusión con profesores, con hombres y mujeres adultas diagnosticados(as) con TDAH en sus infancias y con profesionales de la salud.

Para el presente trabajo se ocupan privilegiadamente el análisis de las dos primeras etnografías mencionadas, junto al de las entrevistas a niños, niñas y cuidadores; y adultos alguna vez diagnosticados. Como forma de poner en común las aproximaciones analíticas, se usaron estrategias que siguen la exploración categorial del análisis de contenido cualitativo (NAVARRO; DÍAZ, 1998; VALLES, 1997), junto a elementos del análisis sociológico del discurso (RUIZ, 2009, 2014) y del análisis situacional (CLARKE et al., 2015) que nos permitieron integrar los distintos materiales y las discusiones que se dieron en el seno del equipo de investigación, poniéndolos en relación con elementos extratextuales concernientes a las lecturas del problema escolar en Chile, basados en análisis de segundo orden y documentos oficiales. Por cierto, se siguieron protocolos éticos en cada paso y se resguardará en el presente artículo y en todo momento la identidad de todas las personas participantes.

\section{El problema de la Escuela en Chile}

En mayo de 2006 irrumpe en Chile la protesta más masiva y sostenida en el tiempo de la posdictadura. Iniciada por la(o)s estudiantes de la educación escolar secundaria, logró

\footnotetext{
${ }^{4}$ Hasta antes de la reforma iniciada en el último periodo presidencial de Michelle Bachelet (11 de marzo $2014-$ 11 de marzo 2018), estos colegios se conocían como "particulares - subvencionados", en tanto, eran administrados por privados a los cuales se les permitía lucrar, aun recibiendo fondos directos del Estado bajo el sistema de vouchers, cuestión que será explicada con mayor detalle en el apartado siguiente. Actualmente, esto se encuentra en mutación pasando de manos de privados a fundaciones sin derecho al lucro. La asignación de subvenciones a la demanda sigue, no obstante, inalterada.

${ }^{5}$ Organizados según los criterios de la Asociación de investigadores de mercado (AIM), 2012.
} 
suscitar el apoyo de hasta el $80 \%$ de la población según cifras del periodo (DOMEDEL; PEÑA y Lillo, 2008). Este nivel de adherencia manifestado en las calles, tomas de escuelas y encuestas de opinión se hallaba vinculado al modo en que, avanzando más allá de las demandas económicas típicas de todos los años escolares y, a través de la impugnación de la Ley Orgánica Constitucional de Enseñanza (LOCE) promulgada por Pinochet pocos días antes de deponer su mandato, se expone el funcionamiento de la educación pública (RADISZCZ, 2009). En efecto, lo que desde la llamada revolución de los pingüinos se empieza a cuestionar es el rol y el lugar que la educación posee en el panorama nacional, formulando el eslogan que será retomado luego en 2011 para el caso universitario: la aspiración a una "educación libre, gratuita y de calidad". Tal masividad, nivel de apoyo y persistencia no puede ser comprendido sin referencia a una suerte de contrato afectivo que había organizado las esperanzas y decepciones de las generaciones criadas en dictadura: la meritocracia (MAYOL et al., 2013).

Durante la década de los 90’s pudo observarse cómo el discurso del mérito se actualiza y consagra. En este sentido, por una parte, es evidente que la historia del mérito es de más larga data en el país, específicamente en oposición a lo que, en este territorio, se conoce como pituto ${ }^{6}$ (BAROZET, 2006). Y, a la vez, es claro que su significado, su posición simbólica y afectiva se resquebraja a partir de aquellas impugnaciones estudiantiles (ARAUJO; MARTUCCELLI, 2015). La historia del mérito, por llamarlo de alguna forma, había organizado modos de comprender la justicia social que, ante el desmantelamiento del proyecto de un estado de garantías sociales que se desarrolla durante la dictadura y se consagra en su continuidad formalmente democrática (GÁRATE, 2012), adquiere un rasgo marcadamente paradójico, en la medida en que aparece como único bien de salvación, a la vez que productor de nuevos sentimientos de injusticia (ARAUJO; MARTUCCELLI, 2012; MAYOL et al., 2013).

Esta paradoja se produce en relación al modelo de relaciones sociales que, descrito como neoliberalismo, hace confluir en la práctica las dimensiones del mérito y el pituto, las cuales se suponían polaridades éticas (ARAUJO, 2016; ARAUJO; MARTUCCELLI, 2012). La escuela y, con ello la educación en general, se ven como único bien de salvación en la misma medida de esta paradoja, pues se concibe en ella un lugar para, a la vez, conseguir credenciales formales y redes informales. Un esfuerzo personal y familiar recubre entonces mérito y pituto a la vez, complejizando las tensiones que se ciernen sobre la escuela (ARAUJO; MARTUCCELLI, 2015; BAROZET, 2006).

\footnotetext{
${ }^{6}$ Entendido como la forma coloquial con que se denomina en Chile a un favor recibido, generalmente, desde una persona con mayor jerarquía que permite gozar de un beneficio al que sería difícil optar de otra forma. Es, de esta forma, un operador de redes informales.
} 
Por una parte, que se defrauden las expectativas concernientes a la valorización del mérito a través de la educación, es inherente al propio sistema educativo, pues mientras más gente accede a títulos escolares, técnicos y profesionales, más éstos se desvalorizan, reproduciendo las diferencias sociales ahora legitimadas como diferencias entre los títulos (BOURDIEU; PASSERON, 2005). A la par que, en tanto la proclamada oferta educativa desplaza el problema del acceso a la educación, la alquimia social opera transmutando diferencias sociales en diferenciales de talento, mérito o esfuerzo (BOURDIEU, 2007). Los títulos se estratifican, las exigencias se refuerzan y las promesas de movilidad social asociadas a la educación formal se alejan (ARAUJO; MARTUCCELLI, 2015).

Sin embargo, por otra parte, aquello que parece implicar una singularidad respecto de Chile, se asocia a la dimensión no oficial de las redes a las que se puede acceder mediante la escuela. En este sentido, el reclamo por la educación pública exponía antes que el incumplimiento de la promesa oficial, el funcionamiento de este contrato siniestro, inconfesado, en tanto la inexistencia de una educación pública, libre, gratuita y de calidad, hace coincidir con la naturalidad del mercado la calidad de la educación con su precio y, de este modo, con las redes que aseguran la reproducción de las clases más allá y más acá del problema de los títulos, es decir, conservando la cadena de favores (ARAUJO; MARTUCCELLI, 2015; RADISZCZ, 2009).

Institucionalmente son la deuda, el copago y el financiamiento a la demanda, a través de los denominados vouchers (INZUNZA, 2009), aquello que delinean las actuales tensiones del sistema educacional chileno, siguiendo de complejas maneras los parámetros del estado subsidiario definido constitucionalmente (ACEITUNO; RADISZCZ, 2016) ${ }^{7}$. Tal cuestión hace material la presión simbólica que, asociada a su funcionamiento informal, organiza las escuelas.

\footnotetext{
${ }^{7}$ Hasta 2017 el sistema escolar (primaria y secundaria) chileno mantenía una macroestructura heredada de las transformaciones dictatoriales de la década del 80 '. Especialmente, a través de: i) la llamada "municipalización" que entregó el control administrativo y financiero a esas unidades territoriales, reproduciendo en la escuela las diferencias en recursos económicos de sus habitantes; y ii) la existencia de un sistema tripartito, en donde, junto a escuelas pertenecientes a los municipios y las pertenecientes a privados (tradicionalmente asociadas a organizaciones religiosas), se desarrolló un sistema mixto, en donde, diversos empresarios se hacen dueños de escuelas, las cuales además reciben apoyo estatal, mediante un subsidio a la demanda o voucher; se les conoce como "particulares-subvencionados" y, teóricamente permitirían, que las familias pudieran elegir libremente en la oferta educativa, fomentando la competencia entre los colegios en calidad. Las protestas estudiantiles expusieron lo que en la práctica se mostró como una pauperización del sector público y una estratificación nuevamente mediada por el poder adquisitivo (ORELLANA, 2010; INZUNZA, 2009; RADISZCZ, 2009). En el segundo periodo de Bachelet, se retomaron algunos elementos, como la demanda de desmuncipalización y la reelaboración del sistema de colegios "subvencionados", dando origen en noviembre de 2017 a la llamada Ley de Nueva Educación Pública que será la transformación más profunda desde 1980, extendiéndose en su implementación hasta 2025; sumándose a la Ley de Inclusión Escolar de 2015 en una amplia modificación de la escuela (Ministerio de Educación (MINEDUC), 2015) A su vez, el actual gobierno de Sebastián Piñera ha aprobado y buscado aprobar otras iniciativas legislativas que apuntan a objetivos divergentes o contrapuestos, lo que sin duda intensifica la tensión que socialmente atraviesa la escuela (BELLEI, 2018).
} 
Éstas, estratificadas en calidad y redes, mediante las sustanciales diferencias en lo que monetariamente pueden aportar las familias (ORELLANA, 2010), se ven consiguientemente pauperizadas basadas en las desigualdades económicas ya existentes y, con ello, se enfrentan a la paradójica e incluso sostenida como imposible demanda de equidad social por la vía educativa (MASCAREÑO, 2005).

La escuela y el sistema educativo en general del que se reclama discursivamente una justicia social que su infraestructura niega en la práctica, se convierte en una potente fuente de ansiedad. Tal tensión explicitada por las protestas sociales desde 2006, se refuerza por los imperativos de inclusión que, siendo contraídos por el Estado con organismos internacionales, comienzan a materializarse desde el año 2008, produciendo una transformación del aula que, al mismo tiempo, dispara los diagnósticos asociados a dificultades conductuales y de aprendizaje, especialmente, el de TDAH (Ministerio de Salud (MINSAL), 2008; VICENTE, SALDIVIA et al., 2012). Pues si bien, desde 1998 comienzan modificaciones en el sistema educativo tendientes a ampliar el curriculum en línea con compromisos internacionales adquiridos (Ministerio de Educación (MINEDUC), 2009b), confirmando una exigencia de universalidad donde reina la segregación; no es hasta 2008 que se promulga la llamada Ley de Subvención Preferencial o Ley SEP (Ministerio de Educación (MINEDUC), 2008) que, reconociendo las múltiples demandas que se ciernen sobre las escuelas, busca gestionar técnicamente la tensión existente.

Intenta responder, pues, por una parte, a los compromisos de tender hacia escuelas inclusivas $\mathrm{y}$, por otra, al reconocimiento de desigualdades intrínsecas entre los establecimientos. No obstante, no modifica el sistema general de financiamiento, sino que lo refuerza. Las escuelas reciben más subvención por cada niña(o) que ingrese en esta subvención preferencial, o sea, aumenta su voucher. En principio esta ley se enfocó en quienes siendo considerados "vulnerables" por ser pobres, requieren mayores recursos para ser educados. En ese sentido, el vínculo entre el reclamo pingüino y el "espíritu” de esta ley es bastante evidente, al igual que su casi nula injerencia en aquello que se propone (ELACQUA, 2012).

No obstante, desde 2009 se establecen criterios complementarios y detallados para, cumpliendo con los compromisos de inclusión, discernir qué tipos de condiciones y/o diagnósticos ingresarían en este sistema preferente bajo el rótulo de Necesidades Educativas Especiales (NEE) (Ministerio de Educación (MINEDUC), 2009a). El resultado es que la(o)s niña(o)s diagnosticada(o)s devienen un atractivo comercial para las escuelas precarizadas, a la par de una creciente intervención sobre los colegios y dentro de las aulas. 
El Estado al entregar más recursos para la gestión de la diversidad en las aulas, exigirá la conformación de un equipo de otros profesionales ${ }^{8}$ debidamente identificado y diferenciado, denominado Programa de Integración Escolar (PIE), encargado dentro y fuera de la sala de clases de atender a niña(o)s que, por diversas razones, hayan sido catalogada(o)s dentro de las NEE. A partir de allí uno de los principales diagnósticos que se dispara en su prevalencia es, justamente, el TDAH. Por esta razón, entre otras, ha ganado importante espacio en la opinión pública la tesis de su sobrediagnóstico, sin embargo, creemos fundamental comprender desde la acción del PIE en el aula el modo en que se hace emerger aquello que denominaremos Situación TDAH (CLARO, 2015; REYES et al., 2019; URIBE et al., 2019), para apreciar el fenómeno en su conjunto. Esta apariencia de sobrediagnóstico es parte de lo que habría que explicar y no una explicación a la prevalencia.

\section{No hay sobre diagnóstico. El fenómeno incluye el exceso}

Los distintos debates y controversias han sido tradicionalmente abordados desde el ángulo de la prevalencia del diagnóstico y de la medicalización del comportamiento infantil, en perjuicio de una perspectiva sobre los usos sociales de éste, de los medicamentos prescritos, y de la experiencia subjetiva junto a las trayectorias sociales de niña(o)s (BEHAGUE; LEZE, 2015; BERGEY et al., 2018; COTTET et al., 2019; ROJAS et al., 2018; URIBE et al., 2019). Ello ha implicado que la atención se centre en la conducta atencional y las consecuencias individuales del diagnóstico, por sobre las experiencias subjetivas y sociales. En este sentido, la idea de un sobrediagnóstico iría de la mano de una problematización ligada a índices de prevalencia, que se centraría en los síntomas individuales, perdiendo de vista aquello que se ha llamado Situación TDAH (COTTET et al., 2019; REYES et al., 2019).

Desde esta perspectiva, lo que nos interesa es aproximarnos, a través del material etnográfico y entrevistas a niña(o)s diagnosticada(o)s y adulta(o)s con diagnóstico en su infancia, a la manera en que se constituye el diagnóstico de déficit atencional con hiperactividad. Comenzaremos desde la descripción etnográfica en las salas de clases de dos

\footnotetext{
${ }^{8}$ El PIE se define como una "estrategia educativa con enfoque inclusivo" para "favorecer la participación y el logro de los objetivos de aprendizaje de todos los estudiantes, aportando recursos y equiparando las oportunidades educativas especialmente para aquellos que presentan mayores necesidades de apoyo para progresar en sus aprendizajes", para lo cual pretende literalmente cambiar el "enfoque desde el déficit a la eliminación de barreras" (Ministerio de Educación (MINEDUC), 2016, p.9). Se constituye así otra aproximación a la "gestión pedagógica" (Ministerio de Educación (MINEDUC), 2016, p.10) para responder a la "diversidad de necesidades de aprendizaje" (Ministerio de Educación (MINEDUC), 2016, p.11), contando para ello con docentes especialistas, profesionales asistentes de la educación y otros profesionales asociados a los ámbitos psicoeducativos y de salud (Ministerio de Educación (MINEDUC), 2016).
} 
escuelas chilenas. Además, cabe destacar que tanto en las entrevistas con niña(o)s y adulta(o)s, se ha establecido sin excepción el punto de partida del diagnóstico en la escuela, la que ha sido referida incluso desde alguna(o)s entrevistada(o)s como "la inquietud escolar", al referirse al foco problemático del diagnóstico en cuestión.

Inicialmente nos detendremos en dos descripciones etnográficas: la primera realizada por una profesora durante su jornada de trabajo a la etnógrafa, sobre una estudiante diagnosticada con TDAH del colegio semi-privado en una comuna de clase media/media baja. La segunda descripción corresponde a la observación del etnógrafo, en el colegio municipal de la comuna de clase media/media-alta:

Le gusta ser el centro de atención. Prefiere hacer lo que quiere. No escucha. No presta mucha atención a los detalles. Se olvida de las cosas. Contesta impulsivamente. Se desorganiza. Está siempre en movimiento. Corre o sube a las bancas cuando sus compañeros están atentos. Interrumpe a las personas (Notas etnografía, Cuaderno de campo 1).

Cuando Antonio busca levantando la mano la participación, algo que sucedió en varias de las clases estudiadas, simplemente no es visto, hasta que Antonio decide responder o participar a viva voz sin respetar el turno de nadie y es, por tanto, reprendido: la profesora le llama la atención (Notas etnografía, Cuaderno de campo 2).

Si miramos las configuraciones discursivas con relación a la atención en el contexto del aula, ésta "se llama" en, al menos, dos sentidos completamente opuestos: una interrupción del orden centrado en el adulto(a) atribuido al niño(a) ("le gusta ser el centro de atención”) y como sanción del adulto profesor(a) al niño(a) ("la profesora le llamó la atención").

La fluctuación de la atención fuera del flujo esperado, es decir, hacia el profesor(a), en las descripciones etnográficas estaría acompañado de la interrupción ("interrumpe a las personas"), la que es descrita de la mano de la disrupción ("contesta impulsivamente. Se desorganiza"), ("participa sin respetar el turno"). Dicha interrupción-disrupción connotaría una aparente falla en el manejo de la propia impulsividad y afectividad (BOWDEN, 2014; CALIMAN, 2008; LAKOFF, 2000) que interrumpe el orden esperado en la sala de clases, tensionando las normas de convivencia establecidas en aquel lugar.

Estas configuraciones discursivas, parecen tratar a la atención como sustancia, como botín, de allí la necesidad de articular la atención a verbos (llamar, acaparar, prestar, poner). Sustancializar la acción de atender, se articularía como una manera de despolitizar la pregunta por el orden y la organización en el aula, convirtiendo la conducta en un déficit y la impulsividad afectiva en una institividad cerebral (EHRENBERG, 2018; MALABOU, 2012). Por ello la ambivalencia de la fórmula "llamar la atención", en tanto conquista y sanción, parece del todo 
oportuna para mostrar una dimensión política que resiste a las configuraciones discursivas adultas que se (re)producen en el aula y que tienden a reducir la pregunta por el orden a la acción de niñas y niños.

En este sentido, nos comenzamos a aproximar a la idea de situación, específicamente Situación TDAH, donde la atención juega un rol central, siendo una suerte de botín en disputa que organiza la condición política de tal situación (COTTET et al., 2019). Es precisamente como lo señala la nota del etnógrafo- la captura de una acción de niña(o)s por parte del profesor(a), a través de la asociación de una conducta con un nombre propio, más allá de los límites de un momento específico, aquello que logra convertir una situación en relación y fluctuante, una situación abierta y compuesta de fuerzas, en una sustancia. La atención entendida como un botín, es decir, un elemento a conquistar, es por ello necesariamente fijada, encuadrada, individualizada y cristalizada.

De ahí que pueda hablarse de déficit. Justamente, las observaciones etnográficas nos muestran que ésta se vuelve problemática cuando niña(o)s se desplazan del flujo atencional esperado en la sala de clases, es decir, cuando distraen la autoridad pedagógica. De este modo, aquello que hemos denominado Situación TDAH expresaría una articulación problemática respecto de las formas en que los impulsos se enrolarían (o no) a lo instituido. En términos de Elias (1990, 1998), el convertir aquel acontecimiento en sustancia sería también un distractor respecto de la (re)producción de la problemática relación entre generaciones, que involucraría precisamente una modulación afectiva en función de órdenes civilizatorios o, dicho en otros términos, una colonización del deseo (BURMAN, 2018; ROLNIK, 2019).

Ahora bien, respecto del modo institucional de abordar aquello que se presenta como problemático bajo la figura del diagnóstico de TDAH, nos aproximaremos a la acción del PIE. Como se ha expuesto, en el sistema escolar chileno el PIE sería por excelencia el dispositivo encargado del trabajo con aquella(o)s estudiantes signada(o)s por el diagnóstico de TDAH, es decir, de la particular gestión de aquello que da nombre a la zona fronteriza que abren la(o)s niña(o)s diagnosticada(o)s: las necesidades educativas especiales (NEE) (Ministerio de Educación [MINEDUC], 2009).

El PIE, busca favorecer la inclusión de estudiantes potenciando sus diferencias, pero no de cualquier manera, sino que haciendo que éstas sean integradas al funcionamiento del aula al modo de la gestión de la diversidad (GAULEJAC; HANIQUE, 2015). Este dispositivo se diferencia de las tareas educativas tradicionales y generales de la escuela, como un subespacio que tiene por objetivo una asistencia individualizada, en paralelo al trabajo del docente, al interior de la sala de clases y en espacios extracurriculares. 
Respecto del funcionamiento del PIE en el aula, resultan elocuentes los siguientes fragmentos descritos por el etnógrafo en el colegio municipal:

Diego comienza a conversar sin poner "atención" a la clase y profesional del PIE, no se demoró en aparecer, señalando que "está descontrolado esta semana".

Los profesores llamarán al orden un par de veces, repartidas en los nombres Claudia y Benjamín, de inmediato entra a la sala una "tía del PIE", quien irá directamente donde Benjamín a darle su medicamento (Notas etnografía, Cuaderno de Campo 2).

En primer lugar, quisiéramos destacar en ambos casos la acción casi inmediata del profesional PIE incluso, como muestra el primer caso, antes de que el niño recibiera una llamada de atención oficial por parte de la profesora. Al respecto es interesante también, la velocidad en interpretar una acción bastante común ("conversar") a través de una formulación que lo signa como descontrol. En este punto es llamativa la rápida e individualizada asistencia del PIE donde el/la profesional parece tener un conocimiento acabado sobre quién es el niño problema y nótese que se trata de un quién correctamente individualizado, no de un problema que comprometa a otra(o)s o a la escuela, pudiendo ser abordado directamente con un medicamento. Este abordaje individualizado en términos de la relación niña(o)-diagnósticotratamiento, bajo de la lógica de las NEE, insinúa la instalación de un diferencial de déficits individuales susceptibles de ser rentabilizados, donde el PIE pareciera configurarse como un dispositivo dispuesto a servir como ortopedia entre el déficit y la posibilidad de hacer funcionales aquellas características, eliminando barreras para su incorporación sin, necesariamente en la práctica, cuestionar el mecanismo.

El movimiento desde el déficit de la institución escolar que debe recurrir a todo tipo de arreglos para conseguir subvenciones - como, por ejemplo, la rentabilización del (sobre) diagnóstico de niña(o)s, para subsanar la falta de recursos estatales destinados a la educación en Chile - hacia el déficit atencional, puede ser comprendido como una táctica local que individualiza una problemática, sustancializando un déficit que, sin embargo, instalado en el registro de lo pre-individual, es decir, afectivo e institucional al mismo tiempo (LARA et al., 2017), excede a cualquier individuo en cuestión (LIEBERT, 2018). Aquel modo de individualizar el déficit enlaza con las exigencias de generar maneras particulares de rendir en la escuela y, por tanto, seguir sosteniéndose arrojada(o)s a una vida en sociedad sin apoyo institucional.

En esta lógica, la acción del PIE se articularía al modo del humanitarismo descrito por Fassin (2016), buscando subsanar de inmediato y a nivel individual cualquier inadecuación 
vinculada a la exigencia y violencia con la que opera la institución. De este modo, se trataría de un dispositivo que, en su búsqueda de integración, pareciera sin querer, (re)situar a los niños y niñas como individuos en déficit, donde al asistir individualmente desconoce y reproduce una violencia escolar y estatal. Ello en la medida que moviliza el problema desde el déficit en la educación en Chile hacia un déficit de niñas y niños individualizados, quienes deben rentabilizar su diferencia o asumir su fracaso. Es decir, la acción del PIE se dirigiría a través de la conducción de la atención de niña(o)s al apaciguamiento de la tensión estructural que se cierne sobre la escuela.

De este modo, toda la atención de una situación que compromete al menos al Estado, la escuela, profesora(e)s, PIE y estudiantes, queda cristalizada en un niño o niña, quien no sólo debe asumir y tratar su déficit, sino que sobre todo debe rentabilizarlo. Al respecto, es relevante ya el clásico argumento de Ilina Singh (2005, 2013, 2014), quien se propone conocer la "perspectiva del niño" indicando que a través de un tratamiento adecuado éste podría no sólo rendir de acuerdo con lo esperado, sino que, mediante un sentimiento de competencia podría generar incluso una imagen de sí mismo(a) más satisfactoria y coherente respecto de sus deseos.

De hecho, Singh concluirá que mientras el tratamiento se encuentre en un marco sociomoral (SINGH, 2007), es decir respecto del cumplimiento de las obligaciones escolares, cuando a niños y niñas se les explica el motivo de éste, lo perciben como una manera de tener mayor libertad sobre sus acciones: "poder elegir" poner más atención, portarse mejor o aumentar el potencial de autorrealización a través del desarrollo del autocontrol (SINGH, 2013, 2014). Es decir, no sólo operaría el imperativo de convertir el déficit en potencial, sino que ello debería causar satisfacción. Este último punto, a partir de las entrevistas a adulta(o)s diagnosticada(o)s con TDAH en su infancia, emerge como un elemento central en sus experiencias: el modo en que aquel déficit ha sido, en los casos "exitosos", rentabilizado en su adultez, a través de sus estudios y trabajos.

Los métodos de este (re)encausamiento no son, como antaño, solo la clásica disciplina escolar o laboral, sino también la rentabilización de estas diferencias en una explotación gozosa (DARDOT; LAVAL, 2009). La superposición o desplazamiento de un disciplinamiento que pretendía solo "normalizar", anulando las diferencias, hacia una gestión que impulsa y motiva su rentabilización, generando las condiciones para su (re)inscripción en las exigencias escolares y luego laborales, no profundiza, sin embargo, el cuestionamiento a la norma que organiza el sistema educacional $\mathrm{y}$, con ello, los imaginarios de justicia social que habían iniciado y sostenido las grandes manifestaciones de 2006 y 2011; por el contrario, parece tender a volverla más inexpugnable, en tanto, debe ser obedecida no para negar una diferencia o diferencial 
portado, sino para hacerse realmente quiénes son. Estas modalidades divergentes de devenir individuos nos lleva a la preguntarnos por el conflicto entre órdenes civilizatorios que atestigua la prevalencia, condiciones y gestión del TDAH en Chile.

\section{Reflexiones finales}

Las condiciones epistémicas de recepción del diagnóstico de déficit atencional, formulado como tal desde la década de los $80^{\prime}$, ponen de relieve su constitución como problema en y por la escuela. De ahí que la comprensión de sus singularidades nacionales inviten a relevar la problematización social de ésta, entendiendo, además, que en ocasiones el lenguaje de la salud mental transporta preocupaciones que exceden el ámbito médico (EHRENBERG, 2010).

En este sentido, las protestas estudiantiles que, irrumpiendo en 2006, se encuentran de diversos modos presentes hasta el día de hoy, apuntan una conflictividad particular de la escuela en Chile. Por una parte, aparecía como un gran articulador del discurso del mérito que, consagrado en la década de los 90, organizaba los imaginarios de justicia social de la generación criada en dictadura. Por otra parte, fue impugnada desde aquellas protestas, no solo por un incumplimiento oficial de las promesas de movilidad social, sino también por sostener las redes informales de favores que históricamente se oponían al registro del mérito (siendo conocido como pituto). Al contrario de lo sostenido en la retórica, el sistema tripartito de educación escolar que pauperizaba las prestaciones públicas no fomentaba un sistema del mérito que rompiera la reproducción de los privilegios mediante el intercambio de favores, sino que tendía a mantenerlo, legitimándolo como libertad de elección de los padres. Tal impugnación organiza hasta nuestros días, en plena transformación del sistema de enseñanza, una serie de dispositivos legales y normativos que intervienen la escuela, en la búsqueda de gestionar técnicamente la impugnación política.

Junto a ello, estos dispositivos portan también la tensión introducida por compromisos internacionales que impulsan la constitución de escuelas más inclusivas. Se refuerzan así las presiones universalistas sobre una infraestructura fuertemente segregada, lo que se traduce en la práctica en la operación de los dispositivos legales y normativos privilegiadamente a nivel individual, es decir, de la(o)s niña(o)s, particularmente en el llamado Programa de Integración Escolar (PIE). Uno de los resultados de esto es que dentro de las aulas el problema atencional tiende a la individualización y sustancialización para su gestión y resolución rápida en vista de las necesidades de escuelas institucional y económicamente precarizadas. Y, sin embargo, podíamos apreciar que el problema atencional puede ser de mejor manera descrito a través de 
la noción de Situación TDAH, en donde las fuerzas que atraviesan la escuela y las aulas, constituyen lo problemático y no tanto los déficits individuales, susceptibles de ser rentabilizados, puestos a funcionar como capacidades especiales para la misma norma que les individualiza.

La noción de Situación TDAH sirve justamente como vía de entrada a un problema en producción, ya no a un diagnóstico o un déficit susceptible de rentabilizar, nuevamente, de manera individual, sino que más bien, un acontecimiento; al mismo tiempo que posibilita el movimiento del orden de lo individual hacia lo singular. Aquella disputada atención se trataría de un botín portador de una potencia fluctuante que no puede ser simplemente borrada, puesto que:

[...] el nivel más básico de la vida, los juegos de codificación y descodificación le dejan lugar al azar que, antes que ser enfermedad, déficit o monstruosidad, es una perturbación en el sistema informativo, una «omisión». En última instancia, la vida es aquello que es capaz de error, de allí su carácter radical. (FOUCAULT, 2007, p.55).

Se abriría así el paso a una política del intersticio, a unas políticas de la errancia que, asumiendo radicalmente la crisis como exposición de un conflicto que siempre estuvo, pueda observar a partir de aquello que se resiste o escapa a la estandarización, nuevas posibilidades para pensar el vínculo entre salud mental y política.

En este sentido, a modo de conclusión con apertura, es posible afirmar que el foco en la noción de atención y su circulación, permite a través de su fuga, devenires y movimientos, abrir el problema desde el dilema de la medicalización en la infancia y de la alta prevalencia del diagnóstico, hacia preguntas en torno a los emplazamientos subjetivos de niñas y niños en situación TDAH; moviéndose del orden del déficit susceptible de rentabilizar, hacía lo que se produce y que no cesa de hacerlo. En este sentido, permite a través de seguir el flujo de atención, mapear nuevas formas de organización en el aula, cuando el orden anterior produce déficit. Es decir, politizar la pregunta por la atención.

Ha sido justamente el material etnográfico el que nos ha permitido desarrollar nuestras interrogantes sobre formas otras respecto de lo instituido para habitar el aula y por otros modos existentes o posibles de gestión de la atención. Al respecto resulta interesante una observación del etnógrafo:

Un mayor número de niños y niñas se aproximaron a mí. Es que venían a desplegar una de las que, luego sabría, era parte de sus actividades favoritas: conversar. Algo así como la grupalidad de un curso, aquello que lo hace ser efectivamente un grupo-curso y no solo un agregado de individuos (...) Antes 
que se constituyera este grupo, ya varios me habían preguntado cuál era mi rol dentro de la sala de clases, a lo que yo respondía como siempre hice: 'mirar y escribir'. Respuesta por supuesto insatisfactoria, que tuvo que ver, en este curso, con la propia constitución del grupo alrededor mío (...) De esta forma, la curiosidad se expresa abiertamente con una actitud más cercana corporalmente (...) Una de las niñas, quien tiene cierta autoridad sobre la palabra, no duda en decirme que 'somos el curso más desordenado'. Ante mi pregunta respecto a qué opinan de eso, responde 'nos gusta, porque somos chorizos'. Otros compañeros y compañeras asienten y complementan: 'los otros cursos son fomes' (Notas etnografía, Cuaderno de Campo 2).

Este fragmento nos envía a distintas dimensiones del problema. En primer lugar, cambia el foco nuevamente desde la idea de un déficit en un orden diferencial al esperado por la escuela y por el mundo adulto, hacia un exceso, esta vez, no sólo de la atención que recae desde el mundo adulto hacia el infante calificado como disruptivo, ni el exceso en términos de la exigencia, sino en el sentido de la multiplicidad de flujos en circulación en el aula. Ello expresa lúdicamente la dimensión de la niñez como un desafío político para la adultez y, en este caso, la tensión sobre la atención de niños y niñas mostraría ser parte de aquel problema. Desde esta perspectiva en el aula escolar no sólo se (re)produciría la problemática intergeneracional de un rendimiento siempre insuficiente o en déficit del chile neoliberal, sino que también una pregunta por las nuevas formas posibles de enrolamiento de los cuerpos que no obedezcan meramente a la rentabilización individualizante de las diferencias. En segundo lugar, desde la propia identificación del grupo como (“el curso más desordenado o chorizos”) resuena la dificultad o resistencia para inscribirse en el orden, en la norma escolar, lo que a nuestro juicio es un fragmento que expresaría un desafío civilizatorio siempre inalcanzable del todo. La infancia acompañará como huella indeleble a la humanidad y su adultez, resto irreductible y en fuga, devenires minoritarios de interrupción política (DELEUZE, 2005; DELEUZE; GUATTARI, 2002). Finalmente, emerge la dimensión de la curiosidad, aquel flujo atencional que se escapa a cualquier intento de cierre, como un impulso que se resiste a la colonización o civilización (BURMAN, 2018; ROLNIK, 2019). De este modo, la sustancialización de la Situación TDAH se configuraría como un modo para administrar ortopédicamente las interrupciones al flujo esperado institucional y la atención es lo que estaría políticamente en juego en el aula, en su fluctuación, conquista y rendimiento, captura y valorización, circulación y fuga.

\section{REFERENCIAS}

ACEITUNO, Roberto; RADISZCZ, Esteban. Chicago Boys. In: ABARZÚA, M.;

ACEITUNO, Roberto; VALENZUELA, R., eds. Chile. Glosario ilustrado del malestar.

Santiago de Chile: Social-Ediciones, 2016, p. 29-30. 
AMERICAN PSYCHIATRIC ASSOCIATION. Diagnostic and statistical manual of mental disorders, 1980.

ARAUJO, Kathya. L'inflexion du Chili. Contribution au débat. In: Problèmes d'Amerique latine, Jg. 3 (102), 95-109. 2016.

ARAUJO, Kathya; MARTUCCELLI, Danilo. Desafíos comunes. Retrato de la sociedad chilena y sus individuos. Santiago: LOM Ediciones, 2012.

ARAUJO, Kathya; MARTUCCELLI, Danilo. La escuela y la cuestión del mérito: reflexiones desde la experiencia chilena. In: Educação e Pesquisa, Jg. 41 (spe), 1503-1520. 2015.

ASOCIACIÓN DE INVESTIGADORES DE MERCADO (AIM). Nuevo modelo de grupos socioeconómicos. Santiago de Chile, 2012.

BAROZET, Emmanuelle. El valor histórico del pituto: clase media, integración y diferenciación social en Chile. In: Revista de Sociología (20), 69-96. 2006.

BÉHAGUE, Dominique; LÉZÉ, Samuel. Shaping the modern child. Genealogies and ethnographies of developmental science. In: Social science \& medicine (1982) (143), 249254. 2015.

BELLEI, Cristián, ed. Nueva Educación Pública. Contexto, contenidos y perspectivas de la desmunicipalización. Santiago de Chile: CIAE-Universidad de Chile, 2018.

BERGEY, Meredith R.; FILIPE, Angela M.; CONRAD, Peter; SINGH, Ilina, eds. Global perspectives on ADHD. Social dimensions of diagnosis and treatment in 16 countries. Baltimore: Johns Hopkins University Press, 2018.

BOURDIEU, Pierre. El sentido práctico. Buenos Aires: Siglo veintiuno, 2007.

BOURDIEU, Pierre; PASSERON, Jean Claude. La reproducción. Elementos para una teoría del sistema de enseñanza. México: Fontamara, 2005.

BOWDEN, Gregory. Disorders of inattention and hyperactivity. In: History of the Human Sciences, Jg. 27 (1), 88-107. 2014.

BRAVO, Luis. Trastornos de aprendizaje y de la conducta escolar. Psicopatología de la disfunción cerebral infantil. Santiago de Chile: Andrés Bello, 1977.

BURMAN, Erica. Fanon, education, and action. Child as method / Erica Burman. London: Routledge, 2018.

BURMAN, Erica. Child as method: implications for decolonising educational research. In: International Studies in Sociology of Education, Jg. 28 (1), 4-26. 2019.

CALIMAN, Luciana Vieira. Os valores da atenção e a atenção como valor. In: Estudos e Pesquisas em Psicologia, Jg. 8 (3), 632-645. (http://www.epublicacoes.uerj.br/index.php/revispsi/article/download/10551/8280). 2008. 
CALIMAN, Luciana Vieira. Os regimes da atenção na subjetividade contemporânea. In: Arquivos Brasileiros de Psicologia, Jg. 64 (1), 2-17. 2012.

CÁMARA DE DIPUTADOS DE CHILE. Boletín 11662-04. Modifica la ley General de Educación y la ley sobre Subvención del Estado a establecimientos educacionales, en el sentido de prohibir que se condicione la permanencia de estudiantes al consumo de medicamentos para tratar trastornos de conducta, 2019.

CLARKE, Adele E.; FRIESE, Carrie; WASHBURN, Rachel, eds. Situational analysis in practice. Mapping research with grounded theory. Walnut Creek California: Left Coast Press, 2015.

CLARO, Sebastián. Luche-Mundo. Una metodología para el abordaje responsable y dialógico del TDAH. In: Estudios pedagógicos (Valdivia), Jg. 41 (especial), 31-50. 2015.

CONRAD, Peter; BERGEY, Meredith R. The impending globalization of ADHD: notes on the expansion and growth of a medicalized disorder. In: Social science \& medicine (1982), Vol. 122, 31-43. 2014.

COTTET, Pablo; BÉLIARD, Aude; NAKAMURA, Eunice. Presentation - Concepts, trajectories, and approaches to "agitation" and "non-conforming children": social and cultural experiences in Brazil, Chile and France. In: Saúde e Sociedade, Jg. 28 (1), 6-11. 2019.

DARDOT, Pierre; LAVAL, Christian. La nouvelle raison du monde. Essai sur la société néolibérale. Paris: Découverte, 2009.

DELEUZE, Gilles. Derrames. Entre el capitalismo y la esquizofrenia. Buenos Aires: Cactus, 2005.

DELEUZE, Gilles; GUATTARI, Félix. Mil mesetas. Capitalismo y esquizofrenia. Valencia: Pre-Textos, 2002.

DOMEDEL, Andrea; PEÑA Y LILLO, Macarena. El mayo de los pingüinos. Santiago de Chile: Radio Universidad de Chile, 2008.

EHRENBERG, Alain. La société du malaise. París: Odile Jacob, 2010.

EHRENBERG, Alain. La mécanique des passions. Cerveau,comportement, société. Paris: Odile Jacob, 2018.

ELACQUA, Gregory. The impact of school choice and public policy on segregation. Evidence from Chile. In: International journal of education development, Jg. 32 (3), 444453. 2012.

ELIAS, Norbert. La sociedad de los individuos. Ensayos. Barcelona: Península, 1990.

ELIAS, Norbert. La civilización de los padres y otros ensayos. Santa Fe de Bogotá, México: Norma, 1998. 
FASSIN, Didier. La razón humanitaria. Una historia moral del tiempo presente. Buenos Aires: Prometeo, 2016.

FOUCAULT, Michel. The archaeology of knowledge and the discourse on language. New York: Barnes \& Noble, 1993.

FOUCAULT, Michel. Nacimiento de la biopolítica. Curso en el Collège de France (19781979). Buenos Aires: Fondo de Cultura Económica, 2007.

GÁRATE, Manuel. La revolución capitalista de Chile. 1973-2003. Chile: Ediciones Universidad Alberto Hurtado, 2012.

GAULEJAC, Vincent de; HANIQUE, Fabienne. Le capitalisme paradoxant. Un système qui rend fou. Paris: Éditions du Seuil, 2015.

INZUNZA, Jorge. La construcción del derecho a la educación y la institucionalidad educativa en Chile. Antecedentes para una polémica de 200 años. Santiago de Chile: Observatorio Chileno de Políticas Educativas (OPECH), 2009.

LAKOFF, Andrew. Adaptative will. The evolution of attention deficit disorder. In: Journal of the History of the Behavioral Sciences, Jg. 36 (2), 149-169. 2000.

LARA, Ali; LIU, Wen; ASHLEY, Colin Patrick; NISHIDA, Akemi; LIEBERT, Rachel Jane; BILLIES, Michelle. Affect and subjectivity. In: Subjectivity, Jg. 10 (1), 30-43. 2017.

LIEBERT, Rachel Jane. Psycurity. Colonialism, Paranoia, and the War on Imagination. Abingdon, Oxon: Routledge, 2018.

MALABOU, Catherine. The new wounded. From neurosis to brain damage. New York: Fordham University Press, 2012.

MASCAREÑO, Aldo. La imposibilidad de la igualdad por la vía educativa. In: Asuntos Públicos (513), Online. 2005.

MAYOL, Alberto; AZÓCAR ROSENKRANZ, Carla; AZÓCAR ORTIZ, Carlos. Eı Chile profundo. Modelos culturales de la desigualdad y sus resistencias. Santiago de Chile: Liberalia Ediciones Ltda, 2013.

MINISTERIO DE EDUCACIÓN (MINEDUC). Ley Subvención Escolar Preferencial. Ley 20.248, 2008.

MINISTERIO DE EDUCACIÓN (MINEDUC). Decreto Fuerza de Ley $\mathbf{N}^{\circ} \mathbf{1 7 0}$. Fija normas para determinar los alumnos con necesidades educativas especiales que serán beneficiarios de las subvenciones para educación especial., 2009a.

MINISTERIO DE EDUCACIÓN (MINEDUC). Decreto Fuerza de Ley $\mathbf{N}^{\circ} \mathbf{2}$. Fija texto refundido, coordinado y sistematizado del decreto con fuerza de ley $\mathbf{n}^{\circ} 2$, de 1996 , sobre subvención del estado a establecimientos educacionales. Chile, 2009b. 
MINISTERIO DE EDUCACIÓN (MINEDUC). Déficit Atencional. Guía para su comprensión y desarrollo de estrategias de apoyo, desde un enfoque inclusivo, en el nivel de Educación Básica. Santiago de Chile: Ministerio de Educación, 2009c.

MINISTERIO DE EDUCACIÓN (MINEDUC). Ley de Inclusión Escolar. Ley 20.845, 2015.

MINISTERIO DE EDUCACIÓN (MINEDUC). Manual de apoyo a la Inclusión Escolar en el marco de la Reforma Educacional. Santiago de Chile: Ministerio de Educación, 2016.

MINISTERIO DE SALUD (MINSAL). Guía Clínica Atención Integral de Niñas/ Niños y Adolescentes con Trastorno Hipercinético/Trastorno de la Atención (THA). Santiago de Chile, Chile, 2008.

MINISTERIO DE SALUD (MINSAL). Programa Nacional de Salud de la Infancia con Enfoque Integral. Santiago de Chile: Ministerio de Salud, 2013.

NAVARRO, Patricio; DÍAZ, Claudio. Análisis de contenido. In: Delgado, Juan Manuel; Gutiérrez, Juan, eds. Métodos y técnicas cualitativas de investigación en ciencias sociales. Madrid: Síntesis, 1998.

ORELLANA, María Isabel. Una mirada a la escuela chilena. Entre lógica y paradoja. Santiago de Chile: Ediciones SM, 2010.

PARENS, Erik; JOHNSTON, Josephine. Facts, values, and attention-deficit hyperactivity disorder (ADHD): an update on the controversies. In: Child and adolescent psychiatry and mental health, Vol. 3, No. 1, 1. 2009.

RADISZCZ, Esteban. Chili 2006. La révolte des Pingouins ou une politique qui ne serait pas de l'homogénéisation. In: Assoun, Paul-Laurent; Zafiropoulos, Markos, eds. Figures cliniques du pouvoir. Paris: Economica-Anthropos, 2009.

REYES, Pablo; COTTET, Pablo; JIMENEZ, Alvaro; JAUREGUI, Gabriela. Rethinking medicalization: discursive positions of children and their caregivers on the diagnosis and treatment of ADHD in Chile. In: Saúde e Sociedade, Jg. 28 (1), 40-54. 2019.

RODRÍGUEZ, Pablo. Las palabras en las cosas. Saber, poder y subjetivación entre algoritmos y biomoléculas. Buenos Aires (Argentina): Cactus, 2019.

ROJAS, Sebastián; ROJAS, Patricio; PEÑA, Mónica. From problematic children to problematic diagnosis. The paradoxical trajcetories of child and adolescent ADHD in Chile. In: Bergey, Meredith R.; Filipe, Angela M.; Conrad, Peter; Singh, Ilina, eds. Global perspectives on ADHD. Social dimensions of diagnosis and treatment in 16 countries. Baltimore: Johns Hopkins University Press, 2018, 310-331.

ROLNIK, Suely. Esferas de la insurrección. Apuntes para descolonizar el inconsciente. Buenos Aires: Tinta Limón, 2019.

RUIZ, Jorge. Análisis sociológico del discurso. Métodos y lógicas. In: Forum: qualitative social research, Jg. 10 (2). 
RUIZ, Jorge. El discurso implícito. Aportaciones para un análisis sociológico / Implicit Discourse: Contributions to a Sociological Analysis. In: Revista Española de Investigaciones Sociológicas. 2014.

SINGH, Ilina. Will the "real boy" please behave: dosing dilemmas for parents of boys with ADHD. In: The American journal of bioethics : AJOB, Vol. 5, No. 3, 34-47. 2005.

SINGH, Ilina. Beyond polemics: science and ethics of ADHD. In: Nature reviews. Neuroscience, Vol. 9, No. 12, 957-964. 2008.

SINGH, Ilina. Not robots. Children's perspectives on authenticity, moral agency and stimulant drug treatments. In: Journal of medical ethics, Vol. 39, No. 6, 359-366. 2013.

SINGH, Ilina. Authenticity, Values, and Context in Mental Disorder: The Case of Children With ADHD. In: Philosophy, Psychiatry, \& Psychology, Jg. 21 (3), 237-240. 2014.

STIGLITZ, Gustavo, ed. DDA, ADD, ADHD, como ustedes quieran. El mal real y la construcción social. Buenos Aires: Grama, 2006.

URIBE, Pía; ABARCA-BROWN, Gabriel; RADISZCZ, Esteban; LÓPEZ-CONTRERAS, Eleonora. ADHD and Gender: subjective experiences of children in Chile. In: Saúde e Sociedade, Jg. 28 (1), 75-91. 2019.

VALLES, Miguel. Técnicas cualitativas de investigación social. Reflexión metodológica y práctica profesional. Madrid: Síntesis, 1997.

VICENTE, Benjamin; LA BARRA, Flora de; SALDIVIA, Sandra; KOHN, Robert; RIOSECO, Pedro; MELIPILLAN, Roberto. Prevalence of child and adolescent psychiatric disorders in Santiago, Chile: a community epidemiological study. In: Social psychiatry and psychiatric epidemiology, Vol. 47, No. 7, 1099-1109. 2012.

VICENTE, Benjamín; SALDIVIA, Sandra; LA BARRA, Flora de; KOHN, Robert; PIHAN, Ronaldo; VALDIVIA, Mario; RIOSECO, Pedro; MELIPILLAN, Roberto. Prevalence of child and adolescent mental disorders in Chile: a community epidemiological study. In: Journal of child psychology and psychiatry, and allied disciplines, Vol. 53, No. 10, 10261035. 2012.

WENDER, Paul H.; TOMB, David A. ADHD. A guide to understanding symptoms, causes, diagnosis, treatment, and changes over time in children, adolescents, and adults. New York NY: Oxford University Press, 2017. 


\section{SOBRE OS AUTORES:}

\section{Hugo Sir}

Doutorado em andamento em Ciências Sociais, Universidad de Chile - Université Paris 8; Instituição do nível mais alto de formação (sigla); Docente Universidad Andrés Bello, Santiago, Chile; Membro do Laboratorio Transdisciplinar en Prácticas Sociales y Subjetividad (LaPSoS). E-mail: hugo.sir.r@gmail.com

iD http://orcid.org/0000-0002-7815-8268

\section{Isis Castañeda}

Mestre em Psicología Clínica de Adultos, Universidad de Chile; Docente da Facultad de Ciencias Universidad Mayor, Santiago, Chile; Membro do Laboratorio Transdisciplinar en Prácticas Sociales y Subjetividad (LaPSoS). E-mail: isis.castaneda.c@gmail.com

(iD) http://orcid.org/0000-0002-3916-0170

\section{Esteban Radiszcz}

Doctor en Psicopatología fundamental y psicoanálisis, Université Paris VII; Docente de graduação e pós-graduação na Universidade do Chile, Santiago, Chile; Membro do Laboratorio Transdisciplinar en Prácticas Sociales y Subjetividad (LaPSoS). E-mail: eradiszcz@ gmail.com (iD) https://orcid.org/0000-0002-3282-6670 